Voix et Images

voixetimages

\title{
Bibliographie de Dany Laferrière
}

\section{Mathieu Lanteigne}

Volume 36, numéro 2 (107), hiver 2011

Dany Laferrière

URI : https://id.erudit.org/iderudit/1002445ar

DOI : https://doi.org/10.7202/1002445ar

Aller au sommaire du numéro

Éditeur(s)

Université du Québec à Montréal

ISSN

0318-9201 (imprimé)

1705-933X (numérique)

Découvrir la revue

Citer ce document

Lanteigne, M. (2011). Bibliographie de Dany Laferrière. Voix et Images, 36(2), 93-112. https://doi.org/10.7202/1002445ar d'utilisation que vous pouvez consulter en ligne.

https://apropos.erudit.org/fr/usagers/politique-dutilisation/ 


\title{
B I B L I G R A PHIE DE DA N Y LAFERRIÈRE
}

\author{
$+++$ \\ MATHIEU LANTEIGNE \\ Université de Moncton
}

\section{E U V R E S}

\section{A. Romans et récits}

+ Comment faire l'amour avec un Nègre sans se fatiguer, Montréal, VLB éditeur, 1985, 155 p. (Rééditions: Paris, Belfond, 1989, 182 p. ; Paris, J'ai lu, 1990, 180 p. ; Paris, Le Serpent à plumes, coll. «Motifs», 1999, 176 p. ; Montréal, TYPO, 2002, 192 p.)

+ Éroshima, Montréal, VLB éditeur, 1987, 168 p. (Réédition: Montréal, TYPO, 1998, 160 p.)

+ L'odeur du café, Montréal, VLB éditeur, 1991, 198 p. (Rééditions: Montréal, TYPO, 1999, 240 p. ; Paris, Le Serpent à plumes, 2001, 226 p.)

+ Le goût des jeunes filles, Montréal, VLB éditeur, 1992, 206 p. (Rééditions : Montréal, VLB éditeur, 2004, 331 p. ; Paris, Grasset, 2005, 400 p.)

+ Cette grenade dans la main du jeune Nègre est-elle une arme ou un fruit?, Montréal, VLB éditeur, 1993, 200 p. (Rééditions: Montréal, TYPO, 2000, 220 p. ; Paris, Le Serpent à plumes, coll. «Fiction française», 2002, 356 p. ; Montréal, VLB éditeur, 2002, 353 p.)

+ Chronique de la dérive douce, Montréal, VLB éditeur, 1994, 136 p.

+ Pays sans chapeau, Outremont, Lanctôt, 1996, 221 p. (Rééditions: Paris, Le Serpent à plumes, coll. «Motifs», 1999, 275 p. ; Outremont, Lanctôt, 1999, 234 p. ; Paris, Le Serpent à plumes, 2004, 275 p. ; Montréal, Boréal, coll. «Boréal compact», 2006, 280 p.)

+ La chair du maître, Outremont, Lanctôt, 1997, 311 p. (Réédition: Paris, Le Serpent à plumes, 2000, 369 p.)

+ Le charme des après-midi sans fin, Outremont, Lanctôt, 1997, 198 p. (Rééditions: Paris, Le Serpent à plumes, 1998, 295 p. ; Montréal, Boréal, coll. «Boréal compact», 2010, 248 p.)

+ Le cri des oiseaux fous, Outremont, Lanctôt, 2000, 318 p. (Rééditions: Paris, Le Serpent à plumes, 2000, 345 p. ; Montréal, Boréal, coll. «Boréal compact», 2010, 347 p.)

+ Je suis fatigué, Vincennes, Les Librairies Initiales, 2000, 44 p. (Rééditions: Outremont, Lanctôt, 2001, 144 p.; Montréal, TYPO, 2005, 232 p.)

+ Vers le sud, Montréal, Boréal, 2006, 256 p. ; Paris, Grasset, 2006, 256 p.

+ Je suis un écrivain japonais, Montréal, Boréal, 2008, 272 p. ; Paris, Grasset, 2008, 270 p. (Réédition: Montréal, Boréal, coll. «Boréal compact», 2009, 272 p.)

+ L'énigme du retour, Montréal, Boréal, 2009, 296 p. ; Paris, Grasset, 2009, 304 p. (Réédition: Montréal, Boréal, coll. «Boréal compact», 2010, 296 p.) 


\section{B. Littérature jeunesse}

+ Je suis fou de Vava, illustrations de Frédéric Normandin, Longueuil, Éditions de la Bagnole, coll. «Taxi», 2006, $44 \mathrm{p}$.

+ La fête des morts, illustrations de Frédéric Normandin, Longueuil, Éditions de la Bagnole, coll. «Taxi», 2009, 42 p.

\section{Articles et textes dans des collectifs, périodiques ou journa $\mathbf{r}$}

+ « Marie Chauvet, amour, colère, folie», Littératures haïtiennes, nº 11, 1983, p. 7-10.

+ «Partiellement nuageux», Le Devoir, 15 novembre 1986, p. E2.

+ «Victime de racisme», La Presse, 15 mai 1987, p. B2.

+ «Paysage d'un peintre primitif», Le Devoir, ${ }^{\text {er }}$ septembre 1990, p. A1 et A3.

+ «Le vert paradis des lectures enfantines», Le Devoir, 9 novembre 1991, p. 13-14.

+ « Noël. Une fois seulement!», La Presse, 20 décembre 1992, p. B1 et B4.

+ "Autoportrait. Laferrière mis à nu...», Qui Hebdo, 19 novembre 1993, p. 38-41.

+ «L'année vue par... », Voir, vol. VIII, n 5, 23 décembre 1993, p. 6.

+ « Jorge Luis Borges lu par Dany Laferrière», Voir, vol. IX, n² 23, 4 mai 1995, p. 8.

+ "La lettre et l'image (correspondance avec Monique Proulx)», Dialogue d'île en île, de Montréal à Haitti, Montréal, Éditions du CIDIHCA/Radio-Canada, 1996, p. 32-52.

+ «Un homme en trois morceaux. Inédit», Tribune juive, vol. XIII, nº 6, août 1996, p. 18-22.

+ "La conquête de l'alphabète», Cahier spécial en alphabétisation: Tout ça, ça se passe quelque part dans l'espace, nº 4, 1997, p. 10-11.

+ «The Heat is on Miami. Bouffée de chaleur», En route, Air Canada, janvier 1997, p. 13-14.

+ «Il est naturel d'aimer ce pays», Le Devoir, 21 juin 1997, p. E6.

+ «First we take Paris... Team Quebec did more than impress in the March 1999 Salon du Livre de Paris - the Paris literary Olympics », traduit du français par David Homel, Weekend Post, 24 avril 1999, p. 14.

+ «Les femmes de ma vie», Châtelaine, vol. XLI, n² 2, février 2000, p. 48-51.

+ «Une journée dans la vie d'un jeune félin», La Presse, 16 avril 2000, p. B7.

+ «Ma découverte du Nouveau Monde», Le Devoir, 31 mars 2001, p. A1.

+ "Comment je suis devenu écrivain", La Presse, 23 avril 2001, p. C3.

+ «Écrire après Blanchot», Magazine littéraire, no 424, octobre 2003, p. 55-56.

+ «Haïti. Le nez de la langue», Magazine littéraire, n 451, mars 2006, p. 47-48.

+ «Dany Laferrière par lui-même», Châtelaine, vol. XLVIII, n 6, juin 2007, p. 111-112.

+ « Je voyage en français», Michel Le Bris et Jean Rouaud (dir.), Pour une littérature-monde, Paris, Gallimard, 2007, p. 87-101.

+ «Lettre à un futur célèbre écrivain», Thomas C. Spear (dir.), Une journée haïtienne, Montréal/Paris, Mémoire d'encrier/Présence africaine, coll. «Chroniques», 2007, p. 239-241.

+ «Un aller simple», Riveneuve Continents, nº 6, août 2008, p. 9-16.

+ «Tout bouge autour de moi», Le Devoir, 23 janvier 2010, p. A6.

+ «Le moment Haïti», Le Devoir, 23 janvier 2010, p. A6.

+ «C'est la question qui importe», Le Monde, 26 mars 2010, p. LIV5.

* Dany Laferrière a tenu une chronique hebdomadaire dans le quotidien La Presse de Montréal de 2002 à 2008. 


\section{Scénarios de film}

+ Comment faire l'amour avec un Nègre sans se fatiguer, Jacques W. Benoît (réal.), texte de Dany Laferrière, Montréal, Stock Films International, 1989, 100 min.

+ Le goût des jeunes filles, John L'Écuyer (réal.), texte de Dany Laferrière, Westmount, Christal Films, 2004, $88 \mathrm{~min}$.

\section{E. Fil m}

+ Comment conquérir l'Amérique en une nuit, Montréal, Boréal Films, 2004, 96 min. (Publication du texte: Outremont, Lanctôt, 2004, 128 p. ; réédition: Montréal, Boréal, coll. «Boréal compact», 2010, 150 p.)

\section{F. A u tres}

+ Les années 80 dans ma vieille Ford, Montréal, Mémoire d'encrier, coll. «Chroniques», 2005, 196 p.

+ Tout bouge autour de moi, Montréal, Mémoire d'encrier, coll. «Chroniques», 2010, 159 p.

\section{G. Entretiens}

+ KHALO, Michel, «Dany Laferrière: Comment faire une entrevue avec un Nègre en se fatiguant», $R G$, février 1986, p. 24-25.

+ DUMAS, Pierre-Raymond, «Entretien avec Dany Laferrière», Conjonction, n 170-171, juilletdécembre 1986, p. 80-81.

+ HOMEL, David, «Interview: Dany Laferrière», Books in Canada, January-February 1988, p. 3738.

+ MARCOTTE, Hélène, «Je suis né comme écrivain à Montréal », Québec français, n 79, automne 1990, p. 80-81.

+ DEMERS, Dominique, «Un Haïtien errant», L'Actualité, vol. XVI, nº 13, 1er septembre 1991, p. 44-51.

+ RICHER, Anne, «Fuir les pièges, les carcans. Anne Richer rencontre... Dany Laferrière», La Presse, 15 mars 1993, p. A1-A2.

+ BORDELEAU, Francine, "Dany Laferrière sans arme et dangereux», Lettres québécoises, n 73 , printemps 1994, p. 9-10.

+ BABIN, Denise, «Entrevue avec Dany Laferrière. Les mots pour le dire», Alternatives, vol. XLI, $\mathrm{n}^{\circ}$ 2, octobre 1997, p. 1.

+ PÉAN, Stanley, «Entretien avec Dany Laferrière», Ici, 10 novembre 1997, p. 24.

+ TÉTU DE LABSADE, Françoise (dir.), Littérature et dialogue interculturel, Sainte-Foy, Presses de l'Université Laval, coll. «Culture française d'Amérique», 1997, p. 81-87.

+ BORDELEAU, Francine, "Dany Laferrière, écrivain de la subversion», Spirale, n 158, janvierfévrier 1998, p. 6.

+ SROKA, Ghila, «Dany Laferrière : de la Francophonie et autres considérations... Entrevue avec Dany Laferrière", Tribune juive, vol. XVI, n 5, août 1999, p. 8-16.

+ COATES, Carroll F., «An Interview with Dany Laferrière», Callaloo, vol. XXII, n 4, Fall 1999, p. 910-921.

+ TERVONEN, Taina, «Entretien avec Dany Laferrière. Paris, octobre 1999. Biographie des territoires", Africultures, n² 29, $1^{\text {er }}$ décembre 1999, en ligne: http://www.africultures.com.

+ J'écris comme je vis. Entretien avec Bernard Magnier, Outremont, Lanctôt, 2000, 247 p. ; Lyon, Éditions La passe du vent, 2000, 224 p. 
+ SROKA, Ghila, «Dany Laferrière: Chronique de la dérive douce. Entrevue avec Dany Laferrière, août 1994 », Île en Île, 2000, en ligne: http://www.lehman.cuny.edu/ile.en.ile/. (Repris dans Ghila Sroka, Conversations avec Dany Laferrière, Montréal, Éditions de la Parole Métèque, 2010, p. 37-49.)

+ SROKA, Ghila, «Dany Laferrière : La chair du maître. Entrevue avec Dany Laferrière, 14 mai 1997 ", Ille en Île, 2000, en ligne: http://www.lehman.cuny.edu/ile.en.ile/. (Repris dans Ghila Sroka, Conversations avec Dany Laferrière, Montréal, Éditions de la Parole Métèque, 2010, p. 75-91.)

+ SAINT-ÉLOI, Rodney, «Dany Laferrière: Chronique de la retraite douce», Boutures, vol. I, n 4 , mars-août 2001, p. 4-9, disponible en ligne sur Île en Île : http://www.lehman.cuny.edu/ ile.en.ile/.

+ MILLOT, Pascale, «Le vertige de l'école», Québec science, vol. XLIV, nº 2, octobre 2005, p. 5861.

+ PAYOT, Marianne, «Dany Laferrière ou l'écrit du corps», L'Express, n² 2850, 16 février 2006, p. 61.

+ BÉRUBÉ, Jade, «Les boucles de Vieux Os», La Presse, 19 avril 2009, p. LECTURES3.

+ COUTURE, François, «Dany Laferrière: la vie, telle quelle», Entre les lignes, vol. 6, nº 1, automne 2009, p. 26-27.

+ PETROWSKI, Nathalie, «Dany en trois temps», La Presse, 19 septembre 2009, p. AS24.

+ SROKA, Ghila, "L'énigme du retour: Interview avec Dany Laferrière", Tribune juive, septembre 2009, p. 43-46. (Repris dans Ghila Sroka, Conversations avec Dany Laferrière, Montréal, Éditions de la Parole Métèque, 2010, p.153-163.)

+ ROUSSEAU, Christine, «Entretien Haïti : le témoignage bouleversant de l'écrivain Dany Laferrière", Le Monde, 18 janvier 2010, p. 1.

+ GAUDREAU, Valérie, «Haïti réveille l'Occident», Le Soleil, 22 janvier 2010, p. 11.

+ SROKA, Ghila, Conversations avec Dany Laferrière, Montréal, Éditions de la Parole Métèque, 2010, 217 p.

\section{EEUVRES PARUES EN TRADUCTION}

+ How to Make Love to a Negro, traduction anglaise de David Homel, Toronto, Coach House Press, 1987, 117 p. (Réédition: Londres, Bloomsbury, 1991, 179 p.)

+ Eroshima, traduction anglaise de David Homel, Toronto, Coach House Press, 1991, 87 p.

+ Eroshima, traduction néerlandaise de Chris van de Poel, Leuven, Kritak-Goossens, 1992, 124 p.

+ Vrijen met een neger tot je zwart ziet, traduction néerlandaise de Chris van de Poel, Leuven, Kritak-Goossens, 1992, 129 p.

+ An Aroma of Coffee, traduction anglaise de David Homel, Toronto, Coach House Press, 1993, $171 \mathrm{p}$.

+ Dining with the Dictator, traduction anglaise de David Homel, Toronto, Coach House Press, 1994, $207 \mathrm{p}$.

+ Why Must a Black Writer Write About Sex?, traduction anglaise de David Homel, Toronto, Coach House Press, 1994, 198 p.

+ Come fare l'amore con un negro senza stancarsi, traduction italienne, Pavia, Liber internazionale, coll. «Labirinti », 1995, 160 p.

+ A Drifting Year, traduction anglaise de David Homel, Toronto, Douglas \& McIntyre, 1997, 118 p.

+ Cómo hacer el amor con un negro sin cansarse, traduction espagnole de Lluís Maria Todó, Barcelone, Ediciones Destino, 1997, 176 p. 
+ Down Among the Dead Men, traduction anglaise de David Homel, Toronto, Douglas \& McIntyre, 1997, $212 \mathrm{p}$.

+ Come far l'amore con un negro senza far fatica, traduction italienne de F. Cane, Milan, La Tartaruga edizioni, 2003, 143 p.

+ Come diventare famosi senza far fatica, traduction italienne de Cecilia Bagnoli, Milan, La Tartaruga edizioni, 2004, 342 p.

+ ¿ Esta granada en manos del joven negro es un arma o una fruta?, traduction espagnole de Manuel Serrat Crespo, Barcelone, El Cobre Ediciones, 2004, 329 p.

+ Verso il sud, traduction italienne de Vincenzo Latronico, Milan, La Tartaruga edizioni, 2006, 319 p.

+ Mwen damou pou Vava, traduction en créole haïtien d'Antoine Lyonel Trouillot, Coconut Creek, FL, Educa Vision, 2007, 50 p.

\section{III.PRIX ET D IS TINCTIONS}

+ Prix Carbet de la Caraïbe pour L'odeur du café, 1991.

+ Prix Edgar-l'Espérance pour Le goût des jeunes filles, 1993.

+ Prix Carbet des Lycéens pour Le cri des oiseaux fous, 2000.

+ Prix Gouverneur de la Rosée du Livre et de la Littérature, représentant de la diaspora, ministère de la Culture, Haïti, 2001.

+ Prix du Livre RFO pour Cette grenade dans la main du jeune Nègre est-elle une arme ou un fruit?, 2002.

+ Prix Zénith pour Comment conquérir l'Amérique en une nuit, Festival des Films du Monde de Montréal, 2004.

+ Prix du Gouverneur général du Canada pour Je suis fou de Vava, 2006.

+ Prix Médicis pour L'énigme du retour, 2009.

+ Grand Prix du livre de Montréal pour L'énigme du retour, 2009.

+ Personnalité de l'année 2009 La Presse/Radio-Canada, 2010.

+ Grand Prix littéraire international Metropolis bleu, 2010.

+ Prix des libraires du Québec pour L'énigme du retour, 2010.

+ Doctorat honorifique de l'Université du Québec à Rimouski, 2010.

\section{RÉCEPTION CRITIQUE ET PRINCIPALES ÉTUDES}

\section{A. Monographies}

+ MATHIS-MOSER, Ursula, Dany Laferrière. La dérive américaine, Montréal, VLB éditeur, coll. «Les champs de la culture», 2003, 344 p.

+ VASILE, Beniamin M., Dany Laferrière. L'autodidacte et le processus de création, Paris, L'Harmattan, coll. «Critiques littéraires », 2008, 288 p.

\section{B. Ét u d e s}

+ VASSAL, Anne, «Lecture savante ou populaire: Comment faire l'amour avec un Nègre sans se fatiguer, de Dany Laferrière», Discours social/Social Discourse, vol. II, nº 4, hiver 1989, p. 184202.

+ VASSAL, Anne, "Racisme et doxa. Le cas de Dany Laferrière», Jacques La Mothe (dir.), Les mauvais genres. Actes du colloque organisé au Centre culturel canadien de Paris les 23, 24 et 25 novembre 1989, Liège, Centre de Lecture publique de la Communauté française, coll. «Les cahiers des paralittératures», 1989, p. 167-178. 
+ BERROUËT-ORIOL, Robert et Robert FOURNIER, «L'émergence des écritures migrantes et métisses au Québec», Québec Studies, vol. 14, Spring/Summer 1992, p. 7-22.

+ PURDY, Anthony G., "Altérité, authenticité, universalité: Dany Laferrière et Régine Robin ", Dalhousie French Studies, vol. 23, Fall-Winter 1992, p. 51-59.

+ NAUDIN, Marie, «Dany Laferrière : Être noir à Montréal», Études canadiennes/Canadian Studies, n 38, juin 1995, p. 47-55.

+ RUPRECHT, Alvina, "“L'Amérique c'est moi" : Dany Laferrière and the Borderless Text ", Alvina Ruprecht et Cecilia Taiana (dir.), The Reordering of Culture: Latin America, the Caribbean and Canada in the Hood, Carleton, Carleton University Press, 1995, p. 251-267.

+ VITELLIO, Joëlle, «Poétiques haïtiennes-québécoises: D. Laferrière, É. Ollivier, G. Étienne», Pierre Laurette et Hans-George Ruprecht (dir.), Poétiques et imaginaires. Francopolyphonie littéraire des Amériques, Paris, L’Harmattan, coll. «Critiques littéraires», 1995, p. 349-359.

+ CLARKE, George Elliott, «Liberalism and Its Discontents: Reading Black and White in Contemporary Québécois Texts », Revue d'études canadiennes/Journal of Canadian Studies, vol. XXXI, n³, automne 1996, p. 59-77.

+ LAMONTAGNE, André, " "On ne naît pas Nègre, on le devient". La représentation de l'autre dans Comment faire l'amour avec un Nègre sans se fatiguer de Dany Laferrière », Québec Studies, vol. 23, Spring/Summer 1997, p. 29-42.

+ LAROCHE, Maximilien, «Les étrangers en littérature québécoise. Compareurs et comparés ou comparants?", Neue Romania, nº 18, 1997, p. 75-80.

+ COLEMAN, Daniel, «How to Make Love to a Discursive Genealogy: Dany Laferrière's Metaparody of Racialized Sexuality ", Daniel Coleman (dir.), Masculine Migrations: Reading the Postcolonial Male in New Canadian Narratives, Toronto, University of Toronto Press, 1998, p. 52-81.

+ L'HÉRAULT, Pierre, «Le je incertain : fragmentations et dédoublements», Voix et Images, vol. XXIII, n 3, printemps 1998, p. 501-514.

+ NEPVEU, Pierre, «La chambre de Belzébuth», Intérieurs du Nouveau Monde. Essais sur les littératures du Québec et des Amériques, Montréal, Boréal, coll. «Papiers collés», 1998, p. 327345.

+ THÉRIEN, Michel, «Conjonctions et disjonctions dans Chronique de la dérive douce de Dany Laferrière ou poésie de la condition immigrante», Bénédicte Mauguière (dir.), Cultural Identities in Canadian Literature/Identités culturelles dans la littérature canadienne, New York, Peter Lang, 1998, p. 173-182.

+ EKOTTO, Frieda, «Shamelessness as a Creative Mechanism in Jean Genet's “Notre-Damedes-Fleurs" and Dany Laferrière's Comment faire l'amour avec un Nègre sans se fatiguer", Esprit créateur, vol. XXXIX, nº 4, hiver 1999, p. 80-89.

+ ESSAR, Dennis, «Time and Space in Dany Laferrière's Autobiographical Haitian Novels", Callaloo, vol. XXII, n 4, Fall 1999, p. 930-946.

+ PROPHÈTE, Jean L., «Dany Laferrière and the Autobiography of Disorderly Past Times", Callaloo, vol. XXII, n 4, Fall 1999, p. 947-949.

+ MATIS-MOSER, Ursula, «Driften zu neuen Ufern: Dany Laferrières Roman Chronique de la dérive douce (1994)», Gudrun M. Grabher et Sonja Bahn-Coblans (dir.), The Self at Risk in English Literature and Other Landscapes. Das Risiko Selbst in der englischsprachigen Literatur und in anderen Bereichen, Innsbruck, Institut für Sprachwissenschaft, 1999, p. 155-162.

+ DE SOUZA, Pascale, "Comment écrire un roman sans se fatiguer. Stratégies perlocutoires d'un bestseller chez Dany Laferrière», Québec Studies, vol. 27, Spring/Summer 1999, p. 62-69. 
+ MATHIS-MOSER, Ursula, "Déplacement continuel, "chronique de la dérive douce". Dany Laferrière et l'écriture de l'“hétérogène" », Béatrice Bagola (dir.), Le Québec et ses minorités. Actes du Colloque de Trèves du 18 au 21 juin 1997 en l'honneur de Hans-Josef Niederehe, Tübingen, Max Niemeyer Verlag, 2000, p. 157-171.

+ MATHIS-MOSER, Ursula, «L'odeur du café von Dany Laferrière: Im "Paradiesgarten" der Adoleszenz», Ursula Mathis-Moser et al. (dir.), Blumen und andere Gewächse des Bösen, Vienne, Peter Lang, 2000, p. 295-311.

+ MiRAgLIA, Anne Marie, «Dany Laferrière, l'identité culturelle et l'intertexte afro-américain », Présence francophone, n 54, 2000, p. 121-139.

+ DESLAURIERS, Pierre, «African Magico-Medicine at Home and Abroad: Haitian Religious Traditions in a Neocolonial Setting; The Fiction of Dany Laferrière and Russell Banks », Jamie S. Scott et Paul Simpson-Housley (dir.), Mapping the Sacred: Religion, Geography and Postcolonial Literatures, Amsterdam, Rodopi, 2001, p. 337-353.

+ FIGUEIREDO, Eurídice, «Répéter les clichés? Représentations d’Haïti et du Brésil dans la littérature migrante du Québec», Thamyris/Intersecting : Place, Sex and Race, vol. VIII, 2001, p. 223-234.

+ MATHIS, Ursula, «Vers un nouveau baroque. Interférences génériques dans l'œuvre de Dany Laferrière », Jean Cléo Godin (dir.), Nouvelles écritures francophones. Vers un nouveau baroque?, Montréal, Presses de l'Université de Montréal, coll. «Espace littéraire», 2001, p. 216-230.

+ BERNIER, Silvie, «Dany Laferrière : le principe de distinction», Les héritiers d'Ulysse. Essai, Outremont, Lanctôt, 2002, p. 51-82.

+ CHARTIER, Daniel, «Les origines de la littérature migrante. L'immigration littéraire au Québec au cours des deux derniers siècles», Voix et Images, vol. XXVII, n 2, hiver 2002, p. 303-316.

+ COATES, Carroll F., «In the Father's Shadow : Dany Laferrière and Magloire Saint-Aude», Journal of Haitian Studies, vol. VIII, nº 1, Spring 2002, p. 40-55.

+ BRIÈRE, Éloïse, "Langue d'écriture et transculture. Le cas des francophones des Amériques», Québec Studies, no 33, Spring/Summer 2002, p. 135-148.

+ ALBERT, Christiane, «De l'usage de la provocation dans la littérature. Le cas de Dany Laferrière dans Comment faire l'amour avec un Nègre sans se fatiguer ", Marie-Lyne Piccione (dir.), Voies vers l'extrême, voix de l'extrême. Actes des colloques organisés par le Centre d'études canadiennes de l'Université Michel de Montaigne Bordeaux 3, décembre 1999 et décembre 2001, Pessac, Maison des sciences de l'homme d'Aquitaine, 2003, p. 73-80.

+ BRAZIEL, Jana Evans, «From Port-au-Prince to Montréal to Miami : Trans-American Nomads in Dany Laferrière's Migratory Texts», Callaloo, vol. XXVI, nº 1, Winter 2003, p. 235-251.

+ COLIN-THÉBAUDEAU, Katell, «Dany Laferrière exilé au Pays sans chapeau », Tangence, nº 71, hiver 2003, p. 63-77.

+ BRAZIEL, Jana Evans, «Trans-American Constructions of Black Masculinity: Dany Laferrière, le Nègre, and the Late Capitalist American Racial machine-désirante», Callaloo, vol. XXVI, nº 3, Summer 2003, p. 867-900.

+ MUNRO, Martin, «Ethnography, Exile, and Haitian Literary History in Dany Laferrière's Pays sans chapeau ", Journal of Haitian Studies, vol. IX, n² 2, Fall 2003, p. 74-88.

+ BROWN, Anne, «Le parcours identitaire de Dany Laferrière ou “Mon cœur est à Port-au-Prince, mon esprit à Montréal et mon corps à Miami" ", Studies in Canadian Literature/Études en littérature canadienne, vol. XXVIII, nº 2, 2003, p. 39-58. 
+ DASH, J. Michael, «Vital Signs in the Body Politic. Eroticism and Exile in Maryse Condé and Dany Laferrière», The Romanic Review, vol. XCIV, 2003, p. 309-317.

+ HAIG, Sam, «From Exile to "Errance": Dany Laferrière's Cette grenade dans la main du jeune Nègre est-elle une arme ou un fruit?", Gertrude Aub-Buscher et Beverley Ormerod Noakes (dir.), The Francophone Caribbean Today. Literature, Language, Culture. Studies in Memory of Bridget Jones, Barbados/Jamaica/Trinidad and Tobago, The University of the West Indies Press, 2003, p. 60-81.

+ PESSINI, Alba, «Le stéréotype du Noir chez deux écrivains de la diaspora haïtienne: JeanClaude Charles et Dany Laferrière», Patrizia Oppici (dir.), Stereotipi culturali a confronto nella letteratura caraibica, Bologne, CLUEB, 2003, p. 73-89.

+ REDOUANE, Najib, «Écrivains haïtiens au Québec. Une écriture du dépassement identitaire», Globe. Revue internationale d'études québécoises, vol. VI, nº 1, 2003, p. 43-64.

+ NDIAYE, Christiane, «Laferrière en Legba du grand intérieur rouge », Canadart, n 11, printemps 2004, p. 57-66.

+ BRAZIEL, Jana Evans, «Amour, douleur, folie (Love, Sorrow, Madness) : State Violence and Post-Duvalierist Revenants in Dany Laferrière's Le cri des oiseaux fous ", Journal of Haitian Studies, vol. X, n 2, Fall 2004, p. 95-113.

+ COURCY, Nathalie, «Le goût des jeunes filles de Dany Laferrière. Du chaos à la reconstruction du sens", Présence francophone, n 63, 2004, p. 84-94.

+ ESSAR, Denis F., "Dany Laferrière, "Primitive" Writer. A Haitian Esthetic», Marie-Agnès Sourieau et Kathleen M. Balutansky (dir.), Écrire en pays assiégé. Haïti: Writing Under Siege, Amsterdam/New York, Rodopi, 2004, p. 423-457.

+ LAMONTAGNE, André, «Intertextualité et altérité: Comment faire l'amour avec un Nègre sans se fatiguer de Dany Laferrière ", André Lamontagne (dir.), Le roman québécois contemporain : les voix sous les mots, Montréal, Fides, coll. «Nouvelles études québécoises», 2004, p. 155-179.

+ LUCAS, Raphaël, «Le retour problématique au pays natal: Accords perdus (1987) de Roger Dorsinville et Pays sans chapeau (1997) de Dany Laferrière", Dominique Chancé et Dominique Deblaine (dir.), Entre deux rives, trois continents. Mélanges offerts à Jack Corzani, Pessac, Maison des sciences de l'homme d'Aquitaine, 2004, p. 251-262.

+ BEAUQUIS, Corinne, «De Fanon à Laferrière. Des types en stéréo», Initiales, vol. XX, 2005, p. 3-23.

+ BRAZIEL, Jana Evans, « “C'est moi l'Amérique” : Canada, Haiti and Dany Laferrière's Port-auPrince/Montréal/Miami Textual Transmigrations of the Hemisphere», Comparative American Studies, vol. III, n 1, March 2005, p. 29-46.

+ IRELAND, Susan, «Declining the Stereotype in the Work of Stanley Lloyd Norris, Max Dorsinville and Dany Laferrière», Québec Studies, n 39, Spring/Summer 2005, p. 55-77.

+ MUNRO, Martin, «Master of the New : Tradition and Intertextuality in Dany Laferrière's Pays sans chapeau ", Small Axe: A Caribbean Journal of Criticism, vol. XVIII, September 2005, p. 176-188.

+ HANANIA, Cécile, «De Hiroshima à Éroshima : une érotique de la bombe atomique en forme de haïku selon Dany Laferrière», Voix et Images, vol. XXXI, nº 1, automne 2005, p. 75-87.

+ KOM, Ambroise, «Être immigrant sans galérer : les recettes de Dany Laferrière», Mark Lilleleht, Edris Markward et Ahmed Saber (dir.), North-South Linkages and Connections in Continental and Diaspora African Literatures, Trenton, New Jersey, Africa World Press, 2005, p. 429-440.

+ PESSINI, Alba, «Écritures de la demeure dans la littérature haïtienne contemporaine. JacquesStephen Alexis, Émile Ollivier et Dany Laferrière», La Torre di Babele, vol. III, 2005, p. 141-153. 
+ COURCY, Nathalie, «La traversée du Pays sans chapeau. (Con)fusion des mondes, vérités multiples et identités plurielles", Revue de l'Université de Moncton, vol. XXXVII, nº 1, 2006, p. 225-238.

+ DEN TOONDER, Jeanette, «Espace littéraire et voyage identitaire dans l'écriture migrante au Québec. Ying Chen, Dany Laferrière et Régine Robin », Jean Morency, Jeanette den Toonder et Jaap Lintvelt (dir.), Romans de la route et voyages identitaires, Québec, Nota bene, coll. «Terre américaine», 2006, p. 129-150.

+ GRIMARD-DUBUC, Josée, «Pour une sociocritique de l'écrivain fictif. Intérêt de l'œuvre de Dany Laferrière », Mélanie Carrier et Maude Poissant (dir.), Carrefour de lectures littéraires. États de la jeune recherche littéraire, Québec, Centre de recherche interuniversitaire sur la littérature et la culture québécoises, coll. «Interlignes», 2006, p. 31-45.

+ KEROUAC, Sophie, «Zone de turbulence. Port-au-Prince ou le mouvement perpétuel dans Le goût des jeunes filles de Dany Laferrière », Francophonies d'Amérique, n 21, 2006, p. 83-104.

+ MATHIS-MOSER, Ursula, "J'invente Dany Laferrière, auteur tout court" ou, Dany Laferrière dans les Amériques", Zeitschrift für Kanada-Studien, vol. XXVI, n 49, 2006, p. 61-73.

+ MAYR, Suzette, "Absent Black Women in Dany Laferrière's How to Make Love to a Negro", Canadian Literature, n 188, Spring 2006, p. 31-45.

+ PRUD'HOMME, Nathalie, "Mythes états-uniens et questionnement identitaire dans Cette grenade dans la main du jeune Nègre est-elle une arme ou un fruit? de Dany Laferrière», Daniel Chartier, Véronique Pepin et Chantal Ringuet (dir.), Littérature, immigration et imaginaire au Québec et en Amérique du Nord, Paris, L'Harmattan, coll. «Études transnationales, francophones et comparées», 2006, p. 151-170.

+ ARENTSEN, Maria Fernanda, «Le rôle - complexe - des stéréotypes dans le discours du narrateur migrant de Comment faire l'amour avec un Nègre sans se fatiguer », Dalhousie French Studies, vol. 79, Spring 2007, p. 93-110.

+ AZODO, Ada Uzoamaka, «The Issue Is Race: Gender and Sexuality in Dany Laferrière's North American Autobiography ", Ada Uzoamaka Azodo et Maureen Ngozi Eke (dir.), Gender and Sexuality in African Literature and Film, Trenton, New Jersey, Africa World Press, 2007, p. 91-108.

+ HUMPHRIES, T. Mark, «Révolte dans le sérail: la tradition de résistance sexuelle chez René Depestre et Dany Laferrière», Nouvelles Francographies, vol. I, n 1, septembre 2007, p. 151-168.

+ BENALIL, Mounia, «La carnavalisation du religieux dans la littérature migrante au Québec. L'islamisme de Dany Laferrière», Protée, vol. XXXV, n² 2, automne 2007, p. 95-104.

+ BENALIL, Mounia, «La fictionnalisation de la négritude dans Comment faire l'amour avec un Nègre sans se fatiguer de Dany Laferrière. Ses au-delàs et ses limites ", Studies in Canadian Literature/Études en littérature canadienne, vol. XXXII, n 1, 2007, p. 192-211.

+ MATHIS-MOSER, Ursula, «Le cri des oiseaux fous de Dany Laferrière», Gilles Dupuis et KlausDieter Ertler (dir.), À la carte. Le roman québécois (2000-2005), Frankfurt am Main, Peter Lang, 2007, p. 217-241.

+ MUNRO, Martin, «Dany Laferrière. Master of the New ", Exile and Post-1946 Haitian Literature: Alexis, Depestre, Ollivier, Laferrière, Danticat, Liverpool, Liverpool University Press, coll. «Contemporary French and Francophone Culture», 2007, p. 178-205.

+ SALAÜN, Élise, «Quand le serpent se mord la queue : le désir sexuel du sujet masculin en autofiction dans Les miroirs de mes nuits de Roger Fournier et La chair du maître de Dany Laferrière", Isabelle Boisclair (dir.), Nouvelles masculinités (?): l'identité masculine et ses mises en question dans la littérature québécoise, Québec, Nota bene, 2008, p. 95-116. 
+ RANSOM, Amy J., "Ce Zombi égaré est-il un Haïtien ou un Québécois? Le vaudou chez les écrivains haïtiano-québécois », Canadian Literature, n² 203, Winter 2009, p. 63-83.

+ THIBEAULT, Jimmy, «Pour une géopolitique du sexe : le corps comme objet de pouvoir dans La chair du maître de Dany Laferrière», Daniel Castillo Durante, Julie Delorme et Claudia Labrosse (dir.), Corps en marge : représentation, stéréotype et subversion dans la littérature francophone contemporaine, Ottawa, L'Interligne, coll. «Amarres», 2009, p. 45-62.

+ LEVEL-SCOTT, Corrie, "La vérité troublante de Comment faire l'amour avec un Nègre sans se fatiguer de Dany Laferrière», Monika Boehringer, Kirsty Bell et Hans R. Runte (dir.), Entre textes et images. Constructions identitaires en Acadie et au Québec, Moncton, Institut d'études acadiennes, coll. «Pascal-Poirier», 2010, p. 313-327.

+ WALTER, Roland, «Dance on the Transcultural Hyphen : Dis-Placement and Re-Placement in Contemporary New World African Fiction», Susana Araújo, João Ferreira Duarte et Marta Pacheco Pinto (dir.), Trans/American, Trans/Oceanic, Trans/Lation: Issues in International American Studies, Angleterre, Cambridge Scholars Publishing, 2010, p. 285-296.

+ ROUSSEL-GILLET, Isabelle, "Saisissements et dessaisissements, de La chair du maître de Dany Laferrière à Vers le sud de Laurent Cantet», Cadrage.net, novembre 2010, en ligne : http://www.cadrage.net/dossier/cantet.htm.

\section{Portraits}

1. Textes

+ VANDERVLIST, Harry, «Dany Laferrière», Christian Riegel (dir.), Twenty-First Century Canadian Writers, Detroit, Thomson Gale, 2007, p. 136-141.

+ FORTIN, Marie-Claude, «Dany Laferrière», Enfants Québec, vol. XXII, n³, novembre 2009, p. 13-14.

+ L’HOMME, Christina, «Dany Laferrière, écrivain», GEO, n 374, avril 2010, p. 140.

\section{F il m}

+ RUİZ, Pedro (réal.), La dérive douce d'un enfant de Petit-Goâve, Faits Divers Média, 2009, $90 \mathrm{~min}$.

\section{Comptes rendus et articles de presse}

1. Romans et récits

a) Comment faire l'amour avec un Nègre sans se fatiguer

+ BEAULIEU, Ivanhoe, "Comment lire un roman sans se fatiguer», Le Devoir, 23 novembre 1985, p. 27.

+ MARTEL, Réginald, "Dany Laferrière : Montréal en noir sur rose», La Presse, 30 novembre 1985, p. E3.

+ GAUDREAULT, André, «Un grand sourire noir... avec des crocs», Le Nouvelliste, vol. LXVI, n 34, 7 décembre 1985, p. A13.

+ MARTEL, Réginald, «Dans les revues: Le terrible boulot des taupes de l'édition», La Presse, 9 décembre 1985, p. B8.

+ BOIVIN, Jean-Roch, "Opération à livre ouvert», Montréal ce mois-ci, janvier-février 1986, p. 40.

+ SIMARD, France, "Comment faire l'amour avec un Nègre sans se fatiguer. Un roman, plusieurs premières...", Le Droit, 11 janvier 1986, p. 25. 
+ BERNIER, André, «Des Nègres bien sympathiques», Le Journal de Montréal, $1^{\text {er }}$ février 1986, p. 52.

+ POULIN, Gabrielle, «Comment faire l'amour avec un Nègre sans se fatiguer, de Dany Laferrière.

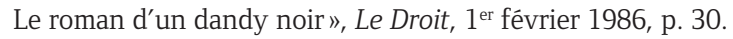

+ LAMY, Suzanne, «Enfin de l'humour noir», Spirale, n 58, février 1986, p. 6.

+ MARCOTTE, Gilles, "Comment faire l'amour avec le lecteur sans se fatiguer», L'Actualité, vol. XI, nº 2, février 1986, p. 126.

+ DÉSILETS, Christian, "Comment faire l'amour avec un Nègre sans se fatiguer", Nuit blanche, no 22, février-mars-avril 1986, p. 5.

+ SROKA, Ghila, "Comment faire l'amour avec un Nègre sans se fatiguer. Dany Laferrière», Tribune juive, vol. III, nº 5, mars-avril 1986, p. 24-26.

+ CLOUTIER, Guy, «L'humour froid et lucide de Laferrière», Le Soleil, 1 ${ }^{\mathrm{er}}$ mars 1986, p. D9.

+ JONASSAINT, Jean, et Anne RACETTE, «L'avenir du roman québécois serait-il métis ? ", Lettres québécoises, no 41, printemps 1986, p. 79-80.

+ JOUAULT, Didier, "Carré blanc. Comment faire l'amour avec un Noir [sic] sans se fatiguer", Nouvelles littéraires, mai 1986, p. 48.

+ PERRON, Gilles, "Comment faire l'amour avec un Nègre sans se fatiguer », Québec français, n 62 , mai 1986, p. 8.

+ SERVET, Michel, «Le repos du dragueur», Jeune Afrique, n 1325, 28 mai 1986, p. 54-55.

+ BEAUDOIN, Réjean, «Les mouches du plafond», Liberté, n 165, juin 1986, p. 126-131.

+ DUMAS, Pierre-Raymond, «Laferrière: comment faire pour écrire nègre + fantasme + blanche?», Conjonction, nos 170-171, juillet-décembre 1986, p. 78-79.

+ PRECOSKY, Don, «Laferrière, Dany: How to Make Love to a Negro», Canadian Book Review Annual, no 13, 1987, p. 130.

+ SIMON, Sherry, "Cherchez le politique dans le roman, en vous fatiguant», Vice Versa, n 17, décembre 1986-janvier 1987, p. 21 et 32.

+ FRASER, Matthew, "Quebec Writer Revels», The Globe and Mail, 4 mars 1988, p. C9.

+ NAIMAN, Sandy, "On A Romp With Love's Dark Side», The Toronto Star, 15 avril 1988, p. 79.

+ TAYLOR, Kate, «Sex Satire», The Spectator, 2 juillet 1988, p. D2.

+ DABBY, Victor, «Controversial novel is published in English», The Telegraph Journal, 9 juillet 1988, s.p.

+ JUBINVILLE, Yves, "Comment faire l'amour avec un Nègre sans jamais [sic] se fatiguer», Continuum, 27 février 1989, p. 22.

+ FOURLANTY, Éric, "Comment faire l'amour avec un Nègre sans se fatiguer. Moi et l'autre», Voir, 2 mars 1989, p. 11.

+ BILOA, Marie-Roger, «Problème : la sexualité comme miroir de l'identité», Jeune Afrique, no 1481, mai 1989, p. 66-68.

+ LENOSKI, Daniel S., «How to Make Love to a Negro by Dany Laferrière and Translated by David Homel», Canadian Literature, nº 120, Spring 1989, p. 236-238.

+ GUAY, Jean, «Sex, jazz et bombe», Québec français, n 79, automne 1990, p. 82-83.

+ SIMON, Sherry, «The Geopolitics of Sex, or Signs of Culture in the Quebec Novel», Essays on Canadian Writing, n 40,1990 , p. 44-49.

+ CAMPBELL, James, «Dining with Miz Literature», Times Literary Supplement, n 4582, 25 janvier 1991, p. 19.

+ ROY, Jean-Hugues, «Le plateau imaginaire. Comme un grand livre ouvert», Voir, vol. VII, nº 12 , 18 février 1993, p. C14. 
+ GREIF, Hans-Jürgen, «La littérature allophone au Québec. Écrire en terre d'accueil», Québec français, $\mathrm{n}^{\circ}$ 105, printemps 1997, p. 61-65.

+ TB, "Comment faire l'amour avec un Nègre sans se fatiguer", Magazine littéraire, n 380, octobre 1999, p. 102.

+ BOIVIN, Aurélien, «Comment faire l'amour avec un Nègre sans se fatiguer ou une dénonciation du racisme à travers la baise», Québec français, n 131, automne 2003, p. 94-97.

+ BREHM, Sylvain, "Dany Laferrière, Comment faire l'amour avec un Nègre sans se fatiguer", Nuit blanche, n 97 , hiver 2004, p. 21.

\section{b ) Éroshima}

+ BARBE, Jean, «Danny [sic] Laferrière en attendant la bombe», Voir, vol. I, nº 33, 13 août 1987, p. 6.

+ LÉVESQUE, Robert, «Bombe et sexe. Le Laferrière nouveau », Le Devoir, 30 septembre 1987, p. 13.

+ PETROWSKI, Nathalie, «En attendant la bombe. Humeurs», Le Devoir, 3 octobre 1987, p. C2.

+ LÉVESQUE, Robert, «Dany Laferrière: “Le talent, ça m'irait!" », Le Devoir, 10 octobre 1987, p. D3.

+ MARTEL, Réginald, «Pétard retentissant et mythe absent», La Presse, 10 octobre 1987, p. J3.

+ ROYER, Jean, «Une bombe dans la tête. Éroshima», Le Devoir, 10 octobre 1987, p. D3.

+ CLOUTIER, Guy, "Éroshima de Laferrière. Sexe et mort: un humour espiègle et insolent», Le Soleil, 7 novembre 1987, p. D8.

+ MONTESSUIT, Carmen, "Éroshima, pas que des fantasmes de Dany Laferrière», Le Journal de Montréal, 22 novembre 1987, p. 33.

+ LAURIN, Michel, «Éroshima. Roman de Dany Laferrière», Nos livres, vol. XIX, nº 1, janvierfévrier 1988, p. 12.

+ VALLIĖRES, Carole, «Dans le blanc des yeux», MTL, janvier-février 1988, p. 12-13.

+ BOIVIN, Jean-Roch, «Vices et vertus de l'exotisme», Lettres québécoises, n 49, printemps 1988, p. 27-29.

+ GUAY, Jean, «Sex, jazz et bombe», Québec français, n 79, automne 1990, p. 82-83.

+ FORAN, Charles, «New Land, New Directions. Writers from Afar Are Making Waves», The Montreal Gazette, 11 mai 1991, p. J1.

+ ROSS, Val, «A Novel and Subversive Use of Raw Fish», The Globe and Mail, 8 juin 1991, p. C6.

\section{c) L'odeur du café}

+ FORTIN, Marie-Claude, «Dany Laferrière. La Mémémoire», Voir, vol. V, n 44, 26 septembre 1991, p. 21.

+ GUAY, Hervé, «Dany Laferrière. Les anciennes odeurs», Le Devoir, 28 septembre 1991, p. D1 et D4.

+ LÉVESQUE, Robert, "Vieux os », Le Devoir, 28 septembre 1991, p. D2.

+ DOSTIE, Bruno, «Dany Laferrière : une parenthèse de bonheur!», La Presse, 29 septembre 1991, p. C1-C2.

+ VOISARD, Anne-Marie, «Le dernier livre de Dany Laferrière : comme si le Québec se retrouvait dans un climat tropical», Le Soleil, 5 octobre 1991, p. F8.

+ VOISARD, Anne-Marie, «L'odeur du café de Dany Laferrière. Un récit tout simple, mais pétri de tendresse et de paix", Le Soleil, 5 octobre 1991, p. F9. 
+ MONTESSUIT, Carmen, «Un autre Dany Laferrière», Le Journal de Montréal, 21 octobre 1991, p. 41.

+ CODINA, Ricardo, "L'odeur du café. Le paradis a un nom: Haïti», Impact Campus, 22 octobre 1991, p. 12.

+ AUCLAIR, Lise, "L'odeur du café. Trop allongé», Continuum, 18 novembre 1991, p. 21.

+ TRUFFAUT, Serge, «La vie littéraire», Le Devoir, 21 décembre 1991, p. D2.

+ JONASSAINT, Jean, «Une histoire d’enfance», Lettres québécoises, n 64, hiver 1991-1992, p. 21.

+ LORTIE, Micheline, «Clip sur le soleil», Femme plus, janvier 1992, p. 8.

+ MARCOTTE, Gilles, «Un automne riche de promesses», L'Actualité, vol. XVII, n 2, $1^{\text {er }}$ février 1992, p. 89.

+ HÉBERT, Pierre, «Éloge de la fiction», Voix et Images, vol. XVII, n 3, printemps 1992, p. 529535.

+ PÉAN, Stanley, «L'odeur du café», Québec français, n 85, printemps 1992, p. 30.

+ CLARKE, George Elliott, «Laferrière Important Canadian Writer», Halifax Chronicle Herald, 20 août 1993, p. B3.

+ BASBANES, Nicholas, «Haitian Memories. A Novel About Dragonflies, Devils and Childhood. An Aroma of Coffee», New York Times Book Review, 28 novembre 1993, p. 15.

+ LAMONT-STEWART, Linda, "An Aroma of Coffee by David Homel and Dany Laferrière», Canadian Literature, n 148, Spring 1996, p. 160-162.

+ ST. PIERRE, Paul Matthew, "L'odeur du café by Dany Laferrière», Canadian Literature, n 148 , Spring 1996, p. 182-184.

+ MOREAU, Gilberte, «L'inscription des valeurs dans L'odeur du café de Dany Laferrière », Québec français, nº 105, printemps 1997, p. 66-69.

\section{d) Le goût des jeunes filles}

+ GAGNON, Katia, "Seules les femmes ont compté pour moi...», La Presse, 8 novembre 1992, p. B1 et B7.

+ ALLARD, Jacques, «Le sourire d'Haïti», Le Devoir, 14 novembre 1992, p. D3.

+ TREMBLAY, Odile, "Dany Laferrière: “Je suis né en riant" ", Le Devoir, 14 novembre 1992, p. D1.

+ FORTIN, Marie-Claude, "Le goût des jeunes filles : des goûts et des couleurs», Voir, vol. VII, $\mathrm{n}^{\circ} 1,26$ novembre 1992, p. 26.

+ ALLARD, Jacques, "Romans québécois», Le Devoir, 19 décembre 1992, p. D8.

+ CONWAY, Daly, «Women have been good to Laferrière», Calgary Herald, 4 janvier 1993, p. B5.

+ MARCOTTE, Gilles, «Un de perdu, un de trouvé », L'Actualité, vol. XVIII, n 4, 15 mars 1993, p. 72.

+ BIRON, Michel, «Du nord au sud», Voix et Images, vol. XVIII, n 3, printemps 1993, p. 610-614.

+ FALARDEAU, Érick, "Le goût des jeunes filles», Québec français, n 89, printemps 1993, p. 28.

+ BORDELEAU, Francine, "Un adolescent en Haïti», Lettres québécoises, nº 70, été 1993, p. 21.

+ DESBIENS-MAgAliOS, Caroline, «Bed Companions», Canadian Literature, n 150, Fall 1996, p. 136-138.

+ MARIN LA MESLÉE, Valérie, «La soif de lire», Magazine littéraire, n 444, juillet-août 2005, p. 70-71.

+ PESSINI, Elena, «Dany Laferrière: Le goût des jeunes filles », Francofonia, vol. XXV, n 49, automne 2005, p. 218-219. 
+ FULTON, Dawn, "Dany Laferrière: Le goût des jeunes filles », The French Review, vol. LXXX, $n^{\circ}$ 6, May 2007, p. 1415-1416.

\section{e) Cette grenade dans la main du jeune Nègre est-elle une arme ou un fruit?}

+ GOYETTE, Danielle, «Les livres qui font... réfléchir», Dernière Heure, nº 9, 1993, p. 58.

+ FORTIN, Marie-Claude, "Cette grenade dans la main du jeune Nègre est-elle une arme ou un fruit? Le système D», Voir, vol. VII, nº 51, 18 novembre 1993, p. 27.

+ ALLARD, Jacques, «Un écrivain mort de rire», Le Devoir, 20 novembre 1993, p. D5.

+ CHARTIER, Jean, «Laferrière dégoupille», Le Devoir, 20 novembre 1993, p. D1-D2.

+ GAGNON, Katia, «Danny [sic] Laferrière à la chasse aux mythes de l'Amérique», La Presse, 21 novembre 1993, p. B1 et B6.

+ VOISARD, Anne-Marie, "Dany Laferrière lance une grenade en plein au ventre de l'Amérique», Le Soleil, 29 novembre 1993, p. B7.

+ HOMIER-ROY, René, « ... et l'Amérique selon Dany », Qui Hebdo, vol. I, nº 10, 3 décembre 1993, p. 39.

+ MONTESSUIT, Carmen, «Pudique comme Dany Laferrière», Le Journal de Montréal, 4 décembre 1993, p. 5.

+ POULIN, Andrée, «Comment devenir célèbre sans se fatiguer», Le Droit, 4 décembre 1993, p. A1-A2.

+ LAFORGE, Christiane, «Dany Laferrière garde le sens du titre », Le Quotidien, 6 décembre 1993, p. 19.

+ CHASSAY, Jean-François, «Topographies américaines», Voix et Images, vol. XIX, n 2, hiver 1994, p. 416-420.

+ L'HEUREUX, Serge, "La vie en Amérique pour Dany Laferrière. Le beau rêve n'est que cauchemar», Le Nouvelliste, 8 janvier 1994, p. P6.

+ FOGLIA, Pierre, «Des livres que je ne lirai pas», La Presse, 17 janvier 1994, p. A5.

+ CRÉPEAU, Jean-François, "Ces états que l'on porte en soi et qui n'ont rien à voir avec ceux de l'âme», Le Canada français, 19 janvier 1994, p. B9.

+ MARCOTTE, Gilles, «L'Alberta devenue personnage mythique», L'Actualité, vol. XIX, n² 21,

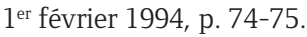

+ JOANISSE, Marc André, «Un départ sans heurts», Le Droit, 24 mars 1994, p. 6.

+ JOANISSE, Marc André, "Grosmaire remplit son sac», Le Droit, 26 mars 1994, p. 6.

+ FALARDEAU, Érick, "Cette grenade dans la main du jeune Nègre est-elle une arme ou un fruit?", Québec français, n 93, printemps 1994, p. 14.

+ BEAUREGARD, Robert, "Cette grenade dans la main du jeune Nègre est-elle une arme ou un fruit?", Nuit blanche, n 55, printemps 1994, p. 36.

+ DESBIENS-MAGALIOS, Caroline, "Bed Companions», Canadian Literature, nº 150, Fall 1996, p. 136-138.

+ MARIN LA MESLÉE, Valérie, «Dany Laferrière : Cette grenade dans la main du jeune Nègre estelle une arme ou un fruit?", Magazine littéraire, n 413, octobre 2002, p. 79.

+ BREHM, Sylvain, «Dany Laferrière: Cette grenade dans la main du jeune Nègre est-elle une arme ou un fruit?", Nuit blanche, nº 91, été 2003, p. 20.

+ SAINT-FORT, Hugues, "Dany Laferrière: Cette grenade dans la main du jeune Nègre est-elle une arme ou un fruit?", The French Review, vol. LXXVII, nº 2, December 2003, p. 422. 
+ GIBEAULT, Stéphan, «Du faux “je" au vrai “jeu"», Spirale, n 194, janvier-février 2004, p. 24-25.

+ DUMONTET, Danielle, «Dany Laferrière: Cette grenade dans la main du jeune Nègre est-elle une arme ou un fruit?», Etudes francophones, vol. XIX, n 1, printemps 2004, p. 206-208.

\section{f) Chronique de la dérive douce}

+ LAURIN, Danielle, «Dany ou la chronique de la dérive douce», L'Actualité, vol XIX, no 13, $1^{\text {er }}$ septembre 1994, p. 60-64.

+ NAVARRO, Pascale, «Les années de plomb : Dany Laferrière», Voir, vol. VIII, n 41, 8 septembre 1994, p. 4.

+ GUAY, Hervé, «Dany Laferrière. L'écrivain qui se dessinait un visage», Le Devoir, 10 septembre 1994, p. D10.

+ MARTEL, Réginald, «Dérive douce: on dirait que l'auteur s'y est mis sans réelle conviction », La Presse, 11 septembre 1994, p. B7.

+ POULIN, Andrée, «Douce dérive de l'arrivant», Le Droit, 15 octobre 1994, p. A10.

+ LONGCHAMPS, Renaud, «Génies, étonnez-moi!», Nuit blanche, n 58, décembre 1994janvier/février 1995, p. 16-17.

+ FALARDEAU, Érick, "Chronique de la dérive douce», Québec français, n 96, hiver 1995, p. 7.

+ LEMIRE, Marc, "Chronique de la dérive douce», Relations, mars 1995, p. 60-61.

+ DESBIENS-MAGALIOS, Caroline, «Bed Companions», Canadian Literature, n 150, Fall 1996, p. 136-138.

\section{g) Pays sans chapeau}

+ LEDUC, Louise, «Retour au pays du caillou au soleil», Le Devoir, 18 mai 1996, p. D1-D2.

+ VILLENEUVE, Marie-Paule, "Dany Laferrière ou le regard de l'exilé», Le Droit, 18 mai 1996, p. A8-A9.

+ MARTEL, Réginald, «Dany Laferrière. L'écrivain-pèlerin au pays sans chapeau », La Presse, 21 mai 1996, p. E4.

+ FOGLIA, Pierre, «Les derniers amateurs», La Presse, 25 mai 1996, p. A5.

+ FOGLIA, Pierre, «L'écrivain sous un manguier», La Presse, 28 mai 1996, p. A5.

+ BERTAIN, Raymond, «Pays sans chapeau, par Dany Laferrière», Voir, vol. X, nº 2, 30 mai 1996, p. 34.

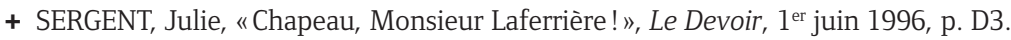

+ DESJARDINS, Sergine, "Dany Laferrière: Pays sans chapeau», Le Fleuve, 22 juin 1996, p. 18.

+ PESANT, Yvon, «Le quotidien de l'Haïti», Le Maskoutain, 18 août 1996, s. p.

+ MARCOTTE, Gilles, «Le retour de l'enfant prodige», L'Actualité, vol. XXI, n 15 , $1^{\text {er }}$ octobre 1996, p. 79-80.

+ BLAISE, Anik, "Pays sans chapeau », Courrier Cadres, n 1296, 16 avril 1999, p. 29.

+ TERVONEN, Taina, «Pays sans chapeau», Africultures, mai 1999, p. 94.

+ NAVARRO, Pascale, «Pays sans chapeau de Dany Laferrière», Voir, vol. XIII, n 26, $1^{\text {er }}$ juillet 1999, p. 48.

+ COATES, Carroll F., «Dany Laferrière: Pays sans chapeau», The French Review, vol. LXXIV, n 4 , March 2001, p. 844-845.

\section{h) La chair du maître}

+ HÉTU, Richard, «La conquête de l'Amérique selon Dany Laferrière», La Presse, 27 avril 1997, p. B1-B2. 
+ FORTIN, Marie-Claude, «La chair du maître. Pouvoir intime», Voir, vol. XI, n 20, 15 mai 1997, p. 38.

+ SERGENT, Julie, "À bride abattue. La chair du maître», Le Devoir, 17 mai 1997, p. D3.

+ MONTESSUIT, Carmen, «Les fruits défendus de Dany Laferrière», Le Journal de Montréal, 18 mai 1997, p. 40.

+ CHARTRAND, Robert, «Dany Laferrière. Haïti, le thêâtre de la séduction», Le Devoir, 31 mai 1997, p. D1-D2.

+ CREVIER, Gilles, «Le sexe dans un kaléidoscope», Le Journal de Montréal, 31 mai 1997, p. 48.

+ SERGENT, Julie, «Terres d'ailleurs. Lectures d'été: deuxième volet», Le Devoir, 21 juin 1997, p. D4.

+ DORION, Gilles, «La chair du maître», Québec français, n 107, automne 1997, p. 15-16.

+ NUOVO, Franco, «Le Salon», Le Journal de Montréal, 19 novembre 1997, p. 53.

+ CRÉPEAU, Jean-François, «Rina Lasnier, La femme furieuse et les autres», Le Canada français, 31 décembre 1997, p. B5.

+ DELAS, Daniel, «Dany Laferrière, La chair du maître», Notre Librairie, n 146 , octobredécembre 2001, p. 91.

+ FULTON, Dawn, "Dany Laferrière: La chair du maître», The French Review, vol. LXXV, n 6, May 2002, p. 1316-1317.

\section{i) Le charme des après-midi sans fin}

+ MARTEL, Réginald, «Les après-midi sans fin de Vieux Os», La Presse, 16 novembre 1997, p. B3.

+ SERGENT, Julie, «Le charme des après-midi sans fin », Voir, vol. XI, n 47, 20 novembre 1997, p. 42.

+ CHARTRAND, Robert, "L'aisance et le labeur. Le charme des après-midi sans fin », Le Devoir, 22 novembre 1997, p. D12.

+ POULIN, Andrée, "Voluptueuses flâneries d'un conteur charismatique », Le Droit, 13 décembre 1997, p. A2.

+ CRÉPEAU, Jean-François, "Offrons des livres et... du pain», Le Canada français, 24 décembre 1997, p. B8.

+ BORDELEAU, Francine, «Le fils de la mémoire», Lettres québécoises, nº 89, printemps 1998, p. 28.

+ DORION, Gilles, "Le charme des après-midi sans fin », Québec français, n 109, printemps 1998, p. 16.

+ LE BEAU, Hélène, «En bref : Le charme des après-midi sans fin », Elle Québec, mars 1998, p. 31.

+ BORDELEAU, Francine, "Soleil trompeur. Le charme des après-midi sans fin», Spirale, n 160 , mai-juin 1998, p. 17.

+ ROY, Monique, "Le charme des après-midi sans fin », Châtelaine, juin 1998, p. 24.

+ CRÉPEAU, Jean-François, «Pour saluer Dany Laferrière», Le Canada français, 22 juillet 1998, p. B7.

+ BÉNICHOU, François, «Le charme des après-midi sans fin», Magazine littéraire, n 369, octobre 1998, p. 126.

+ TERVONEN, Taina, «Le charme des après-midi sans fin», Africultures, mai 1999, p. 94.

+ HADRON-PLANTA, Delta, "Au plaisir de lire les Caraïbes. Dany Laferrière : Le charme des après-midi sans fin», Der Fremdsprachliche Unterricht, vol. XXXIII, nº 42, décembre 1999, p. 4950 . 
+ COATES, Carroll F., «Dany Laferrière : Le charme des après-midi sans fin», The French Review, vol. LXXIV, n 4, March 2001, p. 844-845.

\section{j) Le cri des oiseaux fous}

+ BIRON, Michel, «Lettre à un étudiant», Voix et Images, vol. XXV, n 3, printemps 2000, p. 582587.

+ MARTEL, Réginald, «La cérémonie des adieux», La Presse, 16 mars 2000, p. B3.

+ ROBITAILLE, Louis-Bernard, «Dany Laferrière, le sorcier mégalo», La Presse, 16 mars 2000, p. B3.

+ NAVARRO, Pascale, "Le cri des oiseaux fous», Voir, vol. XIV, nº 12, 23 mars 2000, p. 23.

+ CHARTRAND, Robert, "La mort, l'amour, la vie. Le cri des oiseaux fous », Le Devoir, 25 mars 2000, p. D3.

+ RIOUX, Christian, «Fin de parcours. Dany Laferrière», Le Devoir, 25 mars 2000, p. D1 et D2.

+ LACHANCE, Lise, «Dany Laferrière», Le Soleil, 8 avril 2000, p. D3.

+ BLANCHETTE, Josée, «Haïti chérie. Haïti haïe», Le Devoir, 12 avril 2000, p. A3.

+ LACHANCE, Lise, «La nuit la plus longue», Le Droit, 15 avril 2000, p. A12.

+ MARCOTTE, Gilles, «À chacun son île», L'Actualité, vol. XXV, nº 9, 1"er juin 2000, p. 79-80.

+ CARON, Marie, «Pour une littérature mineure», Lettres québécoises, n 99, automne 2000, p. 25-26.

+ ZAHAR, Christine, "Dany Laferrière. Le cri des oiseaux fous ", Nuit blanche, nº 80, automne 2000, p. 19.

+ COATES, Carroll F., «Dany Laferrière: Le cri des oiseaux fous», The French Review, vol. LXXV, $\mathrm{n}^{\circ}$ 6, mai 2002, p. 1317-1319.

\section{k) Je suis fatigué}

+ PETROWSKI, Nathalie, «Fatigué ? Et moi alors... », La Presse, 26 avril 2001, p. C3.

+ TREMBLAY, Jennifer, «S'épuiser en Amérique», Spirale, nº 181, novembre-décembre 2001, p. 8.

\section{1) Vers le sud}

+ MAGNY, André, «Ces femmes qui achètent du temps», Le Droit, 1 ${ }^{\text {er }}$ avril 2006, p. A18.

+ BROCHU, André, "L'Éden, cash», Lettres québécoises, n 123, automne 2006, p. 20.

+ FULTON, Dawn, «Dany Laferrière: Vers le sud», The French Review, vol. LXXX, n 6, May 2007, p. 1415-1416.

+ HARRIS, Mark, «Rueful affirmative», Canadian Literature, nº 192, Spring 2007, p. 191-192.

\section{m) Je suis un écrivain japonais}

+ LESSARD, Valérie, «Dany Laferrière, écrivain sans frontières», Le Droit, 5 avril 2008, p. A12.

+ ELKOURI, Rima, «Il est un écrivain japonais», La Presse, 6 avril 2008, p. PLUS5.

+ GAUDREAU, Valérie, "Qui suis-je», Le Soleil, 6 avril 2008, p. A6.

+ GUY, Chantal, «Le dernier dandy », La Presse, 6 avril 2008, p. PLUS6.

+ MALAVOY-RACINE, Tristan, "Dany Laferrière. La vérité si je mens», Voir, 10 avril 2008, en ligne : http://www.voir.ca/article.aspx?zone=1\&section=10\&article=57696.

+ DESMEULES, Christian, «L'art poétique de Dany Laferrière», Le Devoir, 12 avril 2008, p. F1.

+ LAURIN, Danielle, "Dany en série», Le Devoir, 12 avril 2008, p. F3.

+ FOGLIA, Pierre, «Trois livres, disons deux et demi», La Presse, 24 avril 2008, p. A7. 
+ PROULX, Paul, «La mondialisation de l'écrivain», Voir, 16 mai 2008, en ligne: http://voir.ca/ publishing $/$ article. aspx?zone $=1 \&$ section $=10 \&$ article $=57696$.

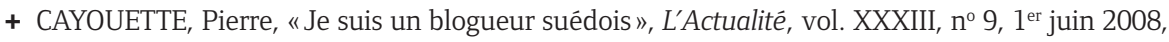
p. 76.

+ LACROIX, Isabelle, «L'identité, l'écriture et la bohème», L'Acadie Nouvelle, 30 août 2008, p. SA2.

\section{n) L'énigme du retour}

+ LAURIN, Danielle, "L'ancien et le nouveau», Le Devoir, 19 septembre 2009, p. F3.

+ LESSARD, Valérie, «Laferrière à sa pleine maturité», Le Droit, 19 septembre 2009, p. A12.

+ MONTPETIT, Caroline, «Des deux côtés de l'exil», Le Devoir, 19 septembre 2009, p. F1.

+ GUY, Chantal, "L'Alpha et l'Omega », La Presse, 5 novembre 2009, p. AS4.

+ LESSARD, Valérie, "La maison de Dany», Le Droit, 7 novembre 2009, p. A2.

+ LAURIN, Danielle, «Les romans québécois de l'année», Le Devoir, 19 décembre 2009, p. F3.

+ BOUCHARD, Marc-André, "Laferrière au pays de son enfance", L'Acadie Nouvelle, 26 décembre 2009, p. SA2.

+ FESSOU, Didier, «Les coups de cœur de l'année», Le Soleil, 27 décembre 2009, p. 33.

+ LAPOINTE, Martine-Emmanuelle, "Le chœur de l'intimité», Voix et Images, vol. 35, nº 2, hiver 2010, p. 120-124.

+ FESSOU, Didier: «Pleins feux sur les livres», Le Soleil, 12 mars 2010, p. 33.

+ CRÉPEAU, Jean-François, «Le récit subversif de Dany Laferrière», Lettres québécoises, n 137 , printemps 2010, p. 26.

+ GAGNON, Laurie, «Dany Laferrière: Les morts dorment au soleil», Le Devoir, $1^{\text {er }}$ mai 2010, p. F2.

+ SOLÉ, Robert, "L'énigme du retour», Le Monde, 2 juillet 2010, p. LIV6.

+ THIBEAUlT, Jimmy, «Le retour d'exil de Windsor Laferrière», Canadian Literature, nº 206, Autumn 2010, p. 154-155.

\section{o) Tout bouge autour de moi}

+ PARÉ, Yvon, «Dany Laferrière ne peut oublier », Lettres québécoises, n 140, hiver 2010, p. 3132.

+ GUY, Chantal, «Un séisme, un livre», La Presse, 26 mars 2010, p. ARTS ET SPECTACLES 3.

+ LAURIN, Danielle, "Dany Laferrière: "Pendant dix secondes, j'ai attendu la mort" ", Le Devoir, 27 mars 2010, p. F3.

+ MONTPETIT, Caroline, "Dany Laferrière - Les dix secondes les plus précieuses de sa vie», Le Devoir, 3 avril 2010, p. F1.

+ TREMBLAY, Odile, "Maux et mots d'Haïti», Le Devoir, 3 avril 2010, p. E2.

+ LESSARD, Valérie, "Tout bouge autour de moi, ou les réflexions intimes de Dany Laferrière», Le Droit, 24 avril 2010, p. A14.

+ LACROIX, Isabelle, «Dany Laferrière dans la tourmente», L'Acadie Nouvelle, 8 mai 2010, p. SA2.

\section{Littérature jeunesse}

\section{a) Je suis fou de Vava}

+ CHAREST, Rémy, "Ailleurs, c'est mieux de l'intérieur», Le Soleil, 14 mai 2006, p. 27.

+ SARFATI, Sonia, «Je suis fou de Vava», La Presse, 16 juillet 2006, p. AS2. 


\section{b) La fête des morts}

+ VOISARD, Anne-Marie, "Quand la mort nous remplit les yeux de papillons», Le Soleil, 20 avril 2009, p. 33.

+ LESSARD, Valérie, «Des papillons dans les yeux des morts», Le Droit, 30 mai 2009, p. A16.

+ MICHAUD, Anne, «Une joyeuse fête des morts», Le Devoir, 30 mai 2009, p. F6.

+ LACROIX, Isabelle, «La beauté de la culture haïtienne», L'Acadie Nouvelle, 11 juillet 2009, p. SA2.

\section{Film}

\section{a) Comment conquérir l'Amérique en une nuit}

+ BRAUTMAN, Davida, «Comment conquérir l'Amérique en une nuit, dir. Dany Laferrière », The French Review, vol. LXXX, n² 2, December 2006, p. 469-470.

\section{Entretiens}

\section{a) J'écris comme je vis}

+ NAVARRO, Pascale, «J'écris comme je vis. Je est un autre», Voir, vol. XIV, n 50, 14 décembre 2000, en ligne: http://www.voir.ca/publishing/article.aspx?zone=1\&section=10\&article=13739.

\section{E. Mémoires et thè ses}

+ AL-HALOOL, Musa, «In Quest of Miranda: Towards a Postcolonial Semantics of Transitive Sex», thèse de doctorat, Pennsylvania State University, 1995, 172 f.

+ COLEMAN, Daniel, «Masculine Migrations: Reading the Postcolonial Male in New Canadian Narratives", thèse de doctorat, University of Alberta, 1995, $353 \mathrm{f}$.

+ BRISEBOIS, Sophie, «Dany Laferrière: parcours d'une écriture», mémoire de maîtrise, Université du Québec à Montréal, 1998, 119 f.

+ ZAHND, Elizabeth A, «Images of the United States in Contemporary Narratives of Quebec and the Francophone Caribbean ", thèse de doctorat, University of Illinois at Urbana-Champaign, 1999, $150 \mathrm{f}$.

+ LABRECQUE, Diane, «Dany Laferrière et Sergio Kokis : la redécouverte de l'Amérique», mémoire de maîtrise, Université du Québec à Montréal, 2001, 100 f.

+ NICOLAS, Lucienne, «Espaces urbains dans le roman de la diaspora haïtienne», thèse de doctorat, Université de Montréal, 2001, 367 f.

+ DUBOIS, Christian, «La raison des passions: les discours de l'identité dans quatre romans d'auteurs immigrants au Québec », mémoire de maîtrise, Université Laval, 2002, 147 f.

+ LEWIS, Daniel, «Pierre Nepveu, Transculturalism and Neo-Quebecois Texts», mémoire de maîtrise, Université de Sherbrooke, 2002, 105 f.

+ BENALIL, Mounia, «La carnavalisation de l'Orient dans la prose narrative francophone (post) moderne (Québec et Maghreb)", thèse de doctorat, University of British Columbia, 2003, $361 \mathrm{f}$.

+ BEAUQUIS, Corinne, «Quatre écrivains haïtiens du Québec: Gérard Étienne, Émile Ollivier, Dany Laferrière et Stanley Péan. Alter-rature ou ubiquité réussie? », thèse de doctorat, The University of Western Ontario, 2005, $358 \mathrm{f}$.

+ SHREAD, Carolyn P. T., «A Theory of Matrixial Reading: Ethical Encounters in Ettinger, Laferriere, Duras and Huston », thèse de doctorat, University of Massachusetts-Amherst, 2005, $314 \mathrm{f}$.

+ GRIMARD-DUBUC, Josée, «Le romancier autofictif : analyse de la représentation du personnage de l'écrivain dans trois romans de Dany Laferrière», mémoire de maîtrise, Université Laval, 2006, 93 f. 
+ DODD, Patrick Lavarte, «The Negro Myth: Ideology of Race, Sexuality and Immobility in Dany Laferrière », thèse de doctorat, University of Michigan, 2007, 160 f.

+ SUBHAGNANA, Xavier, "The Economy of the Migrant Text: Theorizing a French Literature of Migration », thèse de doctorat, University of Wisconsin - Madison, 2007, $209 \mathrm{f}$.

+ WRIGHT, Nancy, «Pre-sent Realities: Counter-Memory in Brand, Clarke, Dorsinville and Laferrière ", mémoire de maîtrise, Université de Sherbrooke, 2007, 88 f.

+ THIBEAULT, Jimmy, «Des identités mouvantes: se définir dans le contexte de la transculturalité», thèse de doctorat, Université d'Ottawa, 2008, 327 f.

+ MATTHEWS, Helen Camp, "Representations of Zombies in Émile Ollivier's La discorde aux cent voix and Dany Laferrière's Pays sans chapeau ", mémoire de maîtrise, University of North Carolina at Chapel Hill, 2009, $30 \mathrm{f}$.

+ GLAUDE, Anne-Marie, «Analyse sémiologique du discours racial en traduction : le Nègre de Dany Laferrière », mémoire de maîtrise, Université Concordia, 2009, 113 f.

+ CLERFEUILLE, Laurence, "Révoltes dans témoin : la tracée du marronnage dans la littérature haïtienne», thèse de doctorat, University of Southern California, 2010, 298 f. 